\title{
A TECHNOLOGY ADAPTATION MODEL FOR BUSINESS PROCESS AUTOMATION
}

\author{
Edward A. Stohr \\ J. Leon Zhao
}

IS-96-14 
Working Paper Series

IS-96-14

\title{
A TECHNOLOGY ADAPTATION MODEL FOR BUSINESS PROCESS AUTOMATION
}

\begin{abstract}
Adapting workflow technologies to support process automation in business organizations is now on the top of the agenda in many organizations. We propose a conceptual model for technology adaptation in this area that stresses both technologyorganization fit and technology-process fit. The goal of our study is to develop a systematic approach to business process automation that is adaptive to changes in organizational needs and is consistent with ideas of worker empowerment. In this paper we suggest a set of fundamental design variables for organizational (organic) and for process (mechanistic) considerations. The technology adaptation model we develop is useful for technology providers in the workflow management area and for business managers who wish to take advantage of the new work-related technologies. The paper concludes with a number of suggestions for future research and development.
\end{abstract}




\title{
A Technology Adaptation Model for Business Process Automation
}

\author{
by \\ Edward A. Stohr \\ Stern School of Business \\ New York University \\ New York, NY \\ estohr@stern.nyu.edu \\ and \\ J. Leon Zhao \\ School of Business and Management \\ The Hong Kong University of Science and Technology \\ Clear Water Bay, Kowloon, Hong Kong \\ zhao@uxmail.ust.hk
}

\begin{abstract}
Adapting workflow technologies to support process automation in business organizations is now on the top of the agenda in many organizations. We propose a conceptual model for technology adaptation in this area that stresses both technology-organization fit and technology-process fit. The goal of our study is to develop a systematic approach to business process automation that is adaptive to changes in organizational needs and is consistent with ideas of worker empowerment. In this paper, We suggest a set of fundamental design variables for organizational (organic) and for process (mechanistic) considerations. The technology adaptation model we develop is useful for technology providers in the workflow management area and for business managers who wish to take advantage of the new work-related technologies. The paper concludes with a number of suggestions for future research and development.
\end{abstract}




\section{Introduction}

The intensity of competition and the complexity and volatility of the business environment is increasing rapidly. As a result, industry and academia have embraced a range of new ideas about organizational structure [Galbraith95] and have turned to business process reengineering as a way of bringing about rapid improvements in the efficiency, responsiveness and flexibility of organizations [Hammer\&Champy93]. These two sets of ideas, the one concerning the need for flatter organizational structures and devolution of decision making authority, and the other concentrating on business processes, are strongly related. Together, they have given rise to a new emphasis on the importance of lateral coordination of activities and to the idea of "process-oriented" organizations [Galbraith95].

Business process reengineering (BPR) involves fundamental changes in the way business activities are organized and executed and is one of the key enablers of the process-oriented view of organizations. According to Earl [Ear194], information technology, enables business process reengineering through its ability to automate, communicate and informate. A broad range of technologies have been developed to support work activities. The application of these technologies to support business processes has been called business process automation (BPA) [Andresen95]. We define BPA as the automation of tasks that were previously performed by humans. This includes activities such as filing and retrieval, physical reproduction and distribution of documents, making and answering telephone calls, manual faxing, and making routine decisions. Thus, BPA is concerned primarily with the automation and communication aspects in Earl's scheme. BPA can be applied to any process whether reengineered or not. Most commentators believe that it is better to reengineer first and then to apply information technology to support the newly designed process [Davenport\&Stoddart94].

Information technologies that support BPA include groupware, e-mail, imaging systems, document management systems (DMS), data warehousing, on-line analytic processing (OLAP), computer telephony integration (CTI), and workflow management systems (WFMS). Related research sub-fields include business process reengineering, computer systems for cooperative work (CSCW), workflow automation, information retrieval, active databases, data mining, decision support systems and organizational learning. All of these technologies and related research areas are devoted to improving and automating the work, and thinking, that is performed within and between business enterprises. While each technology and research substream provides a useful viewpoint, there is a need for integration of the technologies at the technical level and for a better conceptual understanding of how the different perspectives provided by the research subfields can be integrated. 
A second need is to understand how these technologies can be applied to business. Unless care is taken, attempts to automate business processes may have unintended consequences for the organization and may run into obstacles in gaining user acceptance. There is a potential clash between the mechanistic aspects of BPA technologies and the more organic and human aspects of organizations. In particular, it is important to understand how BPA impacts the emerging need for organizations to be adaptable and flexible and to encourage decentralized, innovative thinking when that is called for. This is the central issue of this paper. We propose a framework for considering the broad range of issues that must be addressed in attempting to automate business processes in ways that maintain flexibility and adaptivity and foster rather than inhibit freedom of thought and individual inventiveness. We concentrate on workflow management systems since these integrate the other BPA technologies, place the greatest emphasis on organizational issues of coordination and have the greatest potential to change the nature of work as we know it. Another compelling reason to concentrate on WFMS is that they are now appearing as commercial products being offered by over 50 commercial vendors [Georgakopoulos95]. However, there is little experience in using them and they are not well understood by managers.

The remainder of the paper is organized as follows. In the next section, we provide some background on BPA technologies and WFMS. In section 3, we describe a technology adaptation framework for business process automation that emphasizes the need to recognize the strategic needs of organizations and the multi-faceted nature of work as well as to consider the more mechanistic and rational requirements of specific business processes. In section 4 , we examine general organizational and workrelated needs and identify requirements for adaptability, work flexibility, control, organizational structure and worker empowerment. In section 5, we develop a classification of different process types and a number of metrics and design variables that should be considered when implementing BPA for specific processes. In section 6, we review the design variables developed in the previous two sections and suggest a number of areas for future research. We conclude with a summary and further suggestions for research.

\section{BPA Technologies and Workflow System Concepts}

In this section, we provide some background for the rest of the paper by giving a brief overview of BPA technologies in general and work flow management systems in particular.

From the organizational viewpoint that we are adopting in this paper, work technologies can be divided into three categories as follows:

- Stand-alone: Technologies that can be used independently by individuals. Most first generation office tools such as telephones, fax machines, copiers, spread sheets and word processors fit in this category. 
Newer examples include telephone answering machines, electronic fax, document retrieval systems, mobile telephones and Personal Digital Assistants.

- Collaborative/User Discretion: Technologies in this class are designed to support collaboration and coordination but allow individuals and groups a great deal of freedom in the way that they are adopted and used in the organization. Examples include e-mail, group DSS for meeting support, conference calls, electronic calendering and project management systems, joint document editing and information distribution via the "Intranet".

- Coordinative/User Constraining: Technologies in this group are also designed to support collaboration and coordination but impose strong constraints on the way work is performed in the organization. Work flow management systems are the primary example of technologies in this class. However, other technologies such as EDI, certain types of e-mail system, and groupware such as Lotus Notes when used for work flow can also fall in this cateogry.

It has been found that almost any use of technology in organizations is highly idiosynchratic. Patterns of use and conceptions of the technology emerge through interaction with the human participants in ways that are quite indeterminant [Orlikowski\&Robey91]. This is particularly the case in the second and third categories because of the necessary interaction between individuals. Patterns of use of e-mail and groupware systems (category 2) have been studied fairly extensively (for example, [Sproull\&Kiesler91, Orlikowski92].) Because of their relative newness, relatively few empirical studies have been carried-out in the area of work flow systems. Since WFMS provide less freedom for users, and offer more opportunities for management control, their introduction might be more difficult than that of other groupware.

To set the stage for the remainder of the paper we now provide a brief overview of WFMS. According to the Workflow Management Coalition, an international organization of workflow system vendors, users and analysts, a WFMS is "a system that completely defines, manages and executes workflow processes through the execution of software whose order of execution is driven by a computer representation of the workflow process logic" [WfMC96]. For our purposes, a "work flow system" is an organizational system consisting of humans and computer hardware and software that executes one or more business processes. A WFMS, on the other hand, is a software system that assists in the first, and some or all of the following functions:

1. Routing electronic documents and messages representing tasks to human (or software) agents.

2. Work scheduling - the system maintains "task-lists" to be executed by each agent.

3. Monitoring and controlling workflows in real time.

4. Work load balancing - new tasks may be assigned to agents with the least work on their task lists. 
5. Sending reminders to agents when scheduled tasks are due to be executed.

6. Capturing and reporting the current status of the work system.

7. Recording and reporting the performance of agents.

8. Managing access to document and traditional databases.

9. Interfacing to other systems including imaging systems, mainframe systems, departmental LAN-based systems and remotely located WFMS via VANs.

10.Communicating with the outside world: handling and routing telephone calls, providing fax and paging services and so on.

Many WFMS have been proposed and developed by computer scientists and software developers. A number of features of these systems and a reasonably well-accepted "world-view" of what they entail has begun to emerge [Bussler\&Jablonski94, Kappe195]. In addition, a "reference model" specifying a standardized set of interfaces and data interchange formats has been developed by the Workflow Management Coalition, [WfMC96]. However, many terms are still used interchangeably or defined differently in different systems. In the remainder of the paper we will use the following terms (which are compatible with those in [WfMC96].)

- $\quad$ Agents: In a WFMS, individuals who perform work and coordination functions are referred to as (human) agents. A WFMS may also employ software agents that can perform well defined functions independently of human users.

- Software Tools: It is important to distinguish between software tools (such as word processors) and software agents. Software tools are used directly by humans in the execution of their work while software agents can perform predefined work without agent intervention.

- $\quad$ Roles: An agent can perform one or more roles. For instance, a mechanic may be assigned to car repair and/or to occasional driving duties. However, the roles assigned to each agent must be compatible to its specialties; we refer to the assignment of duties to agents as role assignment.

- Activity: An activity refers to a range of tasks that need to be executed within a business process.

- Task: A task (or work item) is a collection of operations that can be assigned to an agent. A task usually corresponds to a step in a business process. For instance, filling in an invoice transaction.

- Work List: A list of tasks maintained by the WFMS, which is to be executed by an agent.

- Control: Any system must control the access of information, the ways decisions are made, and the quality of work. Controls may take the form of authorization, authentication, verification, and validation. 
- Monitoring: In order to control the quality of work and make policies and rules, the WFMS must monitor the processes and tasks; the monitoring may be done continuously, periodically or on an ad hoc bases.

\section{Business Process Adaptation Model}

BPA Technologies support business processes in the context of the total organization, its strategies, its people, its organizational structure, and its measurement and rewards system. All of these elements need to be jointly designed to ensure the achievement of strategic goals, efficient execution of work, desired behavior of employees and the development of a corporate culture and set of norms that will ensure continued adaptation, innovation and success. These relationships are shown in figure 1 which adds a "BPA Tools" element to the "STAR" organizational design framework of [Galbraith95]. Note that the relationship between BPA technologies and the organization is not only derived from their role in supporting business processes. BPA technologies are used directly by people in the execution of their work and facilitate the monitoring and measurement of performance thereby enabling reward systems that would not be feasible in a paper-based control system.

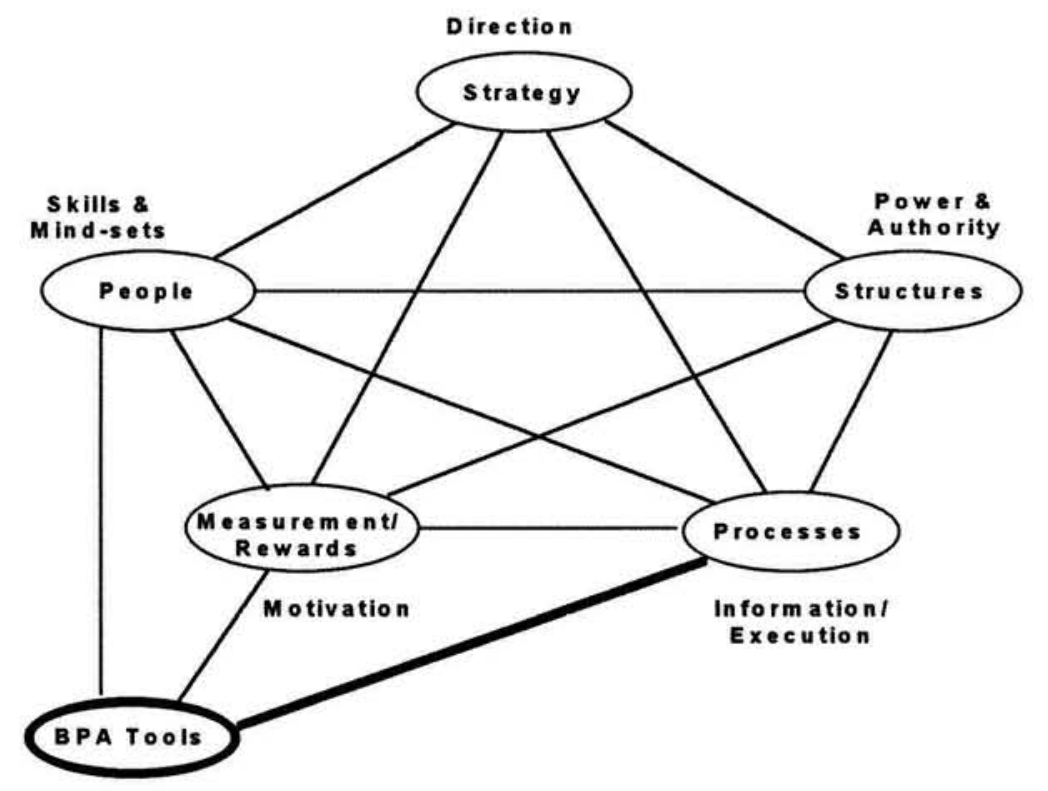

Figure 1. Organizational Context for BPA Implementation

In this paper, we consider the problem of fit between BPA technologies and general organizational and specific process needs. We attempt to characterize these requirements in terms of a number of design variables. Figure 2 illustrates the triangular relationship between the BPA system, the organization, and the processes. According to this model, successful implementation of BPA technologies requires the following: - Process Design Fit: The business process itself must be appropriately designed. 
- Technology-Organization Fit: A good fit of the BPA technologies to the nature of real work and to long-term organizational needs.

- Technology-Process Fit: The chosen BPA technologies must be tailored to the needs of the specific process in which they are embedded.

The use of "adaptation" rather than "fit" in the title for the model emphasizes: (1) the need to adapt whatever BPA technologies are chosen to the nature of the organization and the needs of the particular process, and (2) that the process whereby these technologies are introduced into the organization can take place over a considerable period of time and can be a major determinant of success or failure.

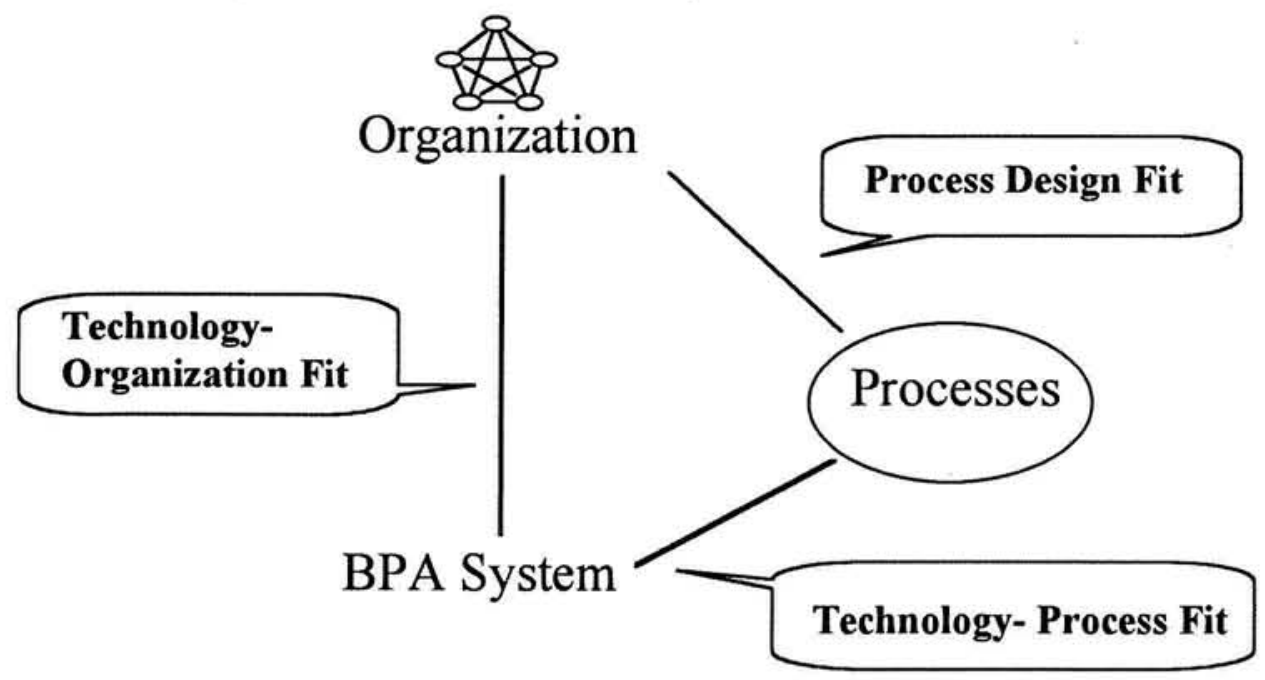

Figure 2. The Triangular Model for BPA Technology Adaptation

Process Design Fit:: To develop a successful implementation of a workflow automation process we will usually need to undertake a reengineering project involving design and implementation steps such as those depicted in Table 1.

\begin{tabular}{|l|l|}
\hline Process Design & Implementation \\
\hline Visioning & Develop IT support \\
\hline Prepare organization for change & Manage change process \\
\hline $\begin{array}{l}\text { Analyze, diagnose and measure existing } \\
\text { processes }\end{array}$ & Implement pilot system and roll-out \\
\hline Determine performance requirements & $\begin{array}{l}\text { Implement performance monitoring and } \\
\text { measurement }\end{array}$ \\
\hline Design new process & (Continuous improvement) \\
\hline $\begin{array}{l}\text { Determine new organizational structure and } \\
\text { design jobs, roles and responsibilities }\end{array}$ & \\
\hline Determine IT requirements & \\
\hline
\end{tabular}

Table 1. Process Design and Implementation Steps 
Note that the determination of IT requirements is the culmination of a number of steps involving the determination of organizational requirements and a subsequent partial redesign of the organization itself. The importance of the steps entitled "preparing the organization for change" and "managing the change process" should also be stressed. Change management turned out to be the single most important implementation problem in a survey of 105 firms who had engaged in reengineering projects [Grovereta195].

In the remainder of this paper, we concentrate on the needs for Technology-Organization (T-O) fit and Technology-Process (T-P) fit. Methods for reengineering business processes to fit organizational needs (as summarized in Table 1), are outside the scope of this paper. However, we develop a classification of the various types of business processes that could be the products of this design process.

Technology-Organization Fit: The T-O dimension emphasizes two conceptually different issues that we feel have been neglected in the application of workflow technologies in practice. The first set of issues arises from the need for these systems to fit the requirements of real work and to support the need for more adaptable, flexible organizational designs. We call this set of issues "organic" in contrast to the purely "mechanistic" approaches that have characterized most workflow system design to date. We believe that mechanistic approaches are needlessly limiting and can lead to implementation failures. The second set of issues concerns the need for BPA technologies to be adopted across the total organization. While the current state of the art probably means that different WFMS will be used for isolated processes, the end result of this could be costly in the same way that non-integrated business data processing systems were limiting and costly in the early days of MIS.

Technology-Process Fit: The T-P dimension focuses on the automation requirements for a specific business process. Each process will have its own specific requirements from both the "organic" and "mechanistic" points-of-view. Once these requirements have been determined an appropriate specification of the work flow system that supports the process can be determined.

Although our research is at a preliminary stage, the identification and classification of workflow system requirements on both the T-O and T-P dimensions should have the following benefits:

1. Provide information for software designers and developers.

2. Improve management understanding of BPA technologies.

3. Provide the basis for more systematic selection and evaluation of BPA hardware and software.

4. Reduce the risks involved in implementing BPA technologies.

In the next two sections, we examine the implications of the T-O and T-P viewpoints for workflow system requirements. 


\section{Technology-Organization Fit: The Clash between Automation and Organizational Needs}

Progress in the development of information technologies makes business process automation an attractive means for increasing organizational efficiency and reducing costs. On the other hand, there is a danger that process automation may institutionalize the "wrong" processes and make the business harder to reengineer in the future. An example of the tendency of automated systems to constrain process innovation is discussed in [Wastelletal94]. The bill production process at a telecommunication services company was well understood and highly automated. However, the handling of exceptional cases, was manual and management wanted to customize the process to individual customer preferences. The design of the data processing system, its rigidity and the difficulties involved in changing it were seen as a major impediment to process improvement. As is the case in most organizations, centralized data processing had achieved huge gains in transaction processing efficiency but had, because of technological limitations and a focus on the main goals of the system at the time of its conception, neglected many secondary information flows and possibilities for variations in the system to fulfill future customer requirements. Mainframe systems have enabled tremendous process improvements (in terms of throughput efficiency and average cycle time reductions). However, the competitive situation today, demands more flexible solutions and more attention to customer service. Considerations of second-order importance at the dawn of the information age are now major issues in an era of increased complexity, speed and competitiveness. Thus, the first issue that must be considered when applying work flow technologies is their impact on organizational adaptability.

\begin{tabular}{|l|l|}
\hline $\begin{array}{l}\text { Organizational/Explicit } \\
\text { (Mechanistic) }\end{array}$ & $\begin{array}{l}\text { Activity-based/Implicit } \\
\text { (Organic) }\end{array}$ \\
\hline Position in hierarchy & Informal politics and network of contacts \\
\hline Definable roles & $\begin{array}{l}\text { Competencies and mutually agreed } \\
\text { responsibilities }\end{array}$ \\
\hline Tasks & Know-how \\
\hline Procedures and techniques & Conceptual understanding \\
\hline Work flow (partial ordering of activities) & Work practices \\
\hline Explicit methods and procedures & Rules of thumb, judgment \\
\hline Electronic documents and messages & $\begin{array}{l}\text { Human communication \& exchange of } \\
\text { experiences }\end{array}$ \\
\hline Teams & Communities \\
\hline Training & Learning \\
\hline
\end{tabular}

Table 2. Mechanistic and Organic Views of Work

A second issue concerns the fundamental assumptions that are made about the nature of work. WFMS research and development efforts have, necessarily, adopted a rather explicit and mechanistic view 
of work in which there are defined set of tasks to be executed by human and software agents and a predetermined partial ordering over the sequence in which these tasks must be executed [Bussler\&Jablonski94]. On the other hand, studies of actual work processes in organizations indicate that work is much more complex and involves the evolution of "work practices" by managers and workers that are continuously adapted and that employ a rich pattern of human communication and sense-making activities. These work practices enable work to be done despite frequent unforeseen exigencies [Suchman83]. These two views of work are summarized in Table 2 which combines two tables from Sachs [Sachs95] and adds several additional issues that we feel are relevant to BPA.

For convenience, we label these two views "mechanistic" and "organic", respectively. Along with [Sachs95], we argue that both views are essential for successful workflow automation. Given the current state of computer science, it is necessary to design work flow management systems according to the mechanistic view. However, to the extent possible, WFMS must accommodate and support the more organic requirements of actual work practice. We call this the requirement for "flexibility" meaning that the WFMS should not unduly restrict users and inhibit the use of common sense and native intelligence that is emphasized by the organic view of work. As an example, [Sachs95] describes the implementation of the Trouble Ticketing System (TTS) at AT\&T. TTS was a large database that also functioned as a scheduling, work routing and record keeping system. Under TTS, workers communicated through the system rather than with each other. "While TTS was designed to make job performance more effective, it had the opposite effect: discouraging the training of new hands, breaking up the community of practice by eliminating trouble-shooting conversations, and extending the amount of time spent on a job by segmenting coherent troubleshooting efforts into unconnected, ticket-based tasks", [Sachs95]. One of the responses of the workers in this case, as in most work situations, was to devise a number of "work-arounds" - ways to get the work done by ignoring or subverting the formal requirements of the system. Work flow systems can never be perfect in practice. A flexible WFMS and workflow design would recognize the inevitability of work-arounds and would, in fact, explicitly encourage them by providing support for user initiatives when exceptions occur. More fundamentally, a flexible WFMS in this case could encourage human conversation by providing easy telephone connections between team members.

Another aspect of work is the interplay between the organizational need for control information and the motivation of employees. BPA technologies provide opportunities for detailed monitoring of performance. However, the desirability for such control, and the type of control that should be used (market-based, bureaucratic or clan-based, [Ouchi79]) varies with the situation. In particular, there is always the choice between measuring performance on the basis of task outcomes or employee conformance 
to prescribed patterns of behavior. As horizontal processes and team-based organizations become more important, there is also a need to collect and measure data at the level of teams rather than individuals. A final dimension of control that is important is the need for high security, integrity and audibility in financial and other processes which might be subject to abuse if not carefully protected.

A third issue concerning workflow systems, involves their compatibility with the objectives and structure of the organization itself. We adopt a simplified view of organizational structure which, nevertheless, is sufficient to determine some of the major requirements for an effective WFMS. By their very nature, WFMS tend to introduce formality and structure into organizations. However, this may not always be desirable. According to the landmark study by Burns and Stalker [Burns\&Stalker 61], when the external environment is stable, firms tend to adopt a "mechanistic" form of internal organization characterized by rules and procedures and a clear hierarchical structure. Internal processes are formalized and centralized with most decisions made at the top. On the other hand, in rapidly changing environments, the internal organization tends to be "organic" with much looser controls, less attention to rules and regulations and a less pronounced hierarchical structure. The mechanistic versus organic dimension can be characterized in terms of the degree of centralization and formalization exhibited by the firm. Centralization is concerned with the delegation of decision authority throughout the organization and the extent of participation by managers in decision making [Aiken\&Hage68]. Formalization is the degree to which rules define roles, authority relations, communications, norms and sanctions and procedures [Halletal67]. Several studies have shown that firms that are more decentralized and less formalized are likely to adapt innovations more rapidly than those that are more structured. For example, an empirical study confirming these findings in the area of adoption of market research results was conducted by Deshpande

[Deshpande82]. Of interest here, are the dimensions used to characterize the perceived structuredness and formality of the organization:

\section{Centralization:}

Participation in Decision Making: the extent and frequency with which lower level managers are consulted and influence decisions.

Hierarchy of Authority: the extent to which authority to make decisions affecting the organization is confined to higher levels.

\section{Formalization:}

Job Codification: the extent to which jobs are rigorously defined

Rule Observation: the degree to which rules are observed and monitored

Job Specificity: the degree to which the specifics of tasks are stated. 
It can be argued that communications capabilities and in particular, groupware, video conferencing and e-mail have the potential either to impose centralized control or to encourage decentralized decision making [Lucas95]. An example of the former is the well-known case of Mrs. Fields Cookies in which management used computer communications capabilities and expert systems to ensure compliance to corporate standards. An example of the latter is given by Frito-Lay in which considerable attention is being given to pushing information and decision making authority down to lower levels in the organization. Whether workflow technologies, can be used in either way is yet to be determined. However, because of their explicit recognition of formal roles and embedded rules, we suspect that most existing WFMS will reinforce tendencies towards centralized decision making. In this context, it should be noted that "rigid hierarchical structures in the organization" was the third most cited cause of BPR failure in the study cited earlier [Groveetal95].

A brief glance at the characterization of formalization given above is sufficient to raise concerns about the efficacy of workflow management systems in situations where innovation, flexibility and managerial intuition and knowledge must be brought to bear on the work situation. All WFMS codify jobs and organizational rules and describe tasks in great specificity. They also tend to enforce the routing of tasks between individuals thereby restricting the free flow of information and flexible collaborative endeavors that are desirable in the newer, more organic forms of organization.

A further potential clash between management requirements and current WFMS capabilities occurs in the area of worker empowerment. As advocated by proponents of lean manufacturing [Womacketal90], total quality management [Pike\&Barnes94] and BPR [Hammer\&Champy94] work empowerment extends beyond simply locating decision making at the level where work is performed. It includes decision making about the design and control of work processes themselves. Under a WFMS, scheduling and work allocation decisions will usually be made by the computer system rather than by individual workers. It may be hard, if not impossible, to allow the workers themselves to control the flow and allocation of work tasks and to design their own work processes.

In the above, we have described several examples of a clash between rigid systems and effective organizational processes. We also observe that unstructured, organic organizational forms are more effective in unstructured situations where knowledge, experience, human collaboration and learning are important. A WFMS should be able to support a formal mechanistic organizational structure in routinizable situations and an organic organizational form with a high degree of worker empowerment in highly unstructured and uncertain situations. Table 3 summarizes our discussion in this section. 


\begin{tabular}{|l|l|}
\hline Organizational Issue/Requirement & Workflow System Design Requirement \\
\hline $\begin{array}{l}\text { 30 Frequent changes in organizational } \\
\text { needs, structure and processes }\end{array}$ & $\begin{array}{l}\text { Adaptability: It should be easy and cheap to adapt the } \\
\text { workflow system as required. }\end{array}$ \\
\hline $\begin{array}{l}\text { The dual mechanistic/organic nature of } \\
\text { work }\end{array}$ & $\begin{array}{l}\text { Work Flexibility: Flexible user environment with strong } \\
\text { communication capabilities, flexible routing schemes. } \\
\text { Control: A flexible range of performance measurement } \\
\text { schemes ranging from none at all to detailed individual or } \\
\text { team performance monitoring. }\end{array}$ \\
\hline $\begin{array}{l}\text { Organizational structure (mechanistic or } \\
\text { organic) }\end{array}$ & $\begin{array}{l}\text { Structure: Capable of representing organizational } \\
\text { structure and locating decisions anywhere in that structure. } \\
\text { Empowerment: The ability of workers to make independent } \\
\text { decisions and to exercise design control over the work } \\
\text { process. }\end{array}$ \\
\hline
\end{tabular}

Table 3. Organizational/Organic Design Variables

For future reference, we refer to the design variables on the right-side of Table 3 as the "AWCSE" variables. The more adaptable the software, the better. The need for the other capabilities (work flexibility, control systems, structure and empowerment) varies with the work situation as discussed below.

Before leaving this section, we mention the issue of user acceptance. There is a fundamental relationship between organization structure, human resources, tasks and reward systems; changing one component implies that adjustments have to be made in the others [Mintzberg79]. BPR efforts often fail because of the difficulties in bringing about change in organizations [Stoddart\&Jarvenpaa95]. Work automation faces the same difficulties. In fact, there is reason to suspect that workflow automation systems are difficult to implement because they fundamentally change the nature of work. Tasks are changed. Communications between workers are altered. The opportunities for casual conversation and direct interaction may be lowered. The pace of work is increased. All of these potential outcomes are likely to be considered dehumanizing. On the positive side, workflow technologies can improve the quality of work life because repetitive and boring tasks can be eliminated. We also argue that proper attention to the adaptability, flexibility and organizational fit issues raised above is a necessary condition for successful WFMS implementation. Many other complex issues involved in a successful WFMS implementation such as preparing the organization for change, training, and developing a culture in which the WFMS is accepted, are outside the scope of this paper. 


\section{Technology-Process Fit: Characterizing Processes and their Support Needs}

In this section, we assume that the process design step has been performed. The task at this stage, is to select various BPA technologies and tailor them to the needs of the specific process. Given the present abundance of WFMS on the market and the lack of experience in using them, this could be a difficult task. As a first step, we attempt to characterize business processes on a number of different dimensions.

Processes that are located differently on these dimensions will have different support needs. In this way, we attempt to build the basis for a more rigorous, contingency-based process for adapting BPA software.

\subsection{AWCSE Requirements of Different Types of Process}

To this point, we have discussed issues of adaptability, work flexibility, control, structure and empowerment at the level of the enterprise. However, different business processes both within and between organizations, will have different requirements along these dimensions.

To illustrate, we define six classes of business process ${ }^{1}$ :

- Accounting Processes: support processes with a major financial component such as purchasing and accounting

- Core processes: value-adding processes that directly satisfy customer needs such as order entry, logistics, claims processing, engineering design and so on.

- Administrative processes: support processes such as time keeping, library services, mailroom and applications for vacation leave.

- Management processes: processes that support the management planning and control function planning, budgeting and performance reporting.

- Knowledge Intensive Processes: processes that gather and process strategic information or special knowledge that represents the core competence of the firm. Examples are processes that support R\&D, market research, and help develop human capital and organizational learning.

- Ad Hoc Processes: processes that satisfy unique, transient needs. Examples are processes to support special staff meetings, small projects, market promotions, conferences and so on.

Note that the first two process types have been automated for many years. The role of WFMS here is to replace paper-based processing, to link support personnel, customers and suppliers more closely and to handle exceptions. Administrative processes have, in the main, been neglected by traditional MIS and

\footnotetext{
${ }^{1}$ Many other classifications of business processes have been proposed, e.g., [Davenport93].
} 
represent a fruitful area ("easy pickings") for cost-cutting BPR applications. Support for management processes has included mainline MIS systems, separate Executive Support Systems (with links to MIS) and OLAP (on-line analytical processing). A new role for WFMS here is primarily document based - supplying executives with correspondence and multimedia documents and enabling communication capabilities such as broadcasts to employees and direct communications with associates and business partners around the world. Knowledge intensive processes have been supported in the past by isolated DSS models and expert systems (for example some accounting firms have embodied their professional knowledge in expert systems for tax assessment). Opportunities for the use of WFMS in this area, include document management and integrated access to data warehouses and organizational learning applications [Stein\&Zwass95]. Finally, ad hoc processes are usually executed intuitively using the learned habits of the responsible groups, supported by mutually agreed agendas and e-mail. There is a need for a workflow management system that can be used by users to develop small project -oriented workflow management systems "on-the-fly".

Table 4 shows possible requirements of each process type in terms of the AWCSE requirements developed in the previous section.

\begin{tabular}{|l|l|l|l|l|l|}
\hline Process Type & Adaptability & Control & $\begin{array}{l}\text { Worker } \\
\text { Flexibility }\end{array}$ & $\begin{array}{l}\text { Formal } \\
\text { Structure }\end{array}$ & $\begin{array}{l}\text { Empower- } \\
\text { ment }\end{array}$ \\
\hline Accounting & Low & High & Low & High & Low \\
\hline Core & Depends & High & Depends & Medium & Depends \\
\hline Administrative & Medium & Medium & Medium & High & Medium \\
\hline Management & Medium & Medium & Medium & Medium & High \\
\hline $\begin{array}{l}\text { Knowledge } \\
\text { intensive }\end{array}$ & Medium & Low & Medium & Low & High \\
\hline Ad hoc & High & Low & High & Low & High \\
\hline
\end{tabular}

Table 4. Characterization of Classes of Business Process

The AWCSE design variables should be evaluated carefully whenever a workflow system is designed. The values in the body of the table are meant to be suggestive only. We have used "depends" to characterize the requirements of core processes to emphasize the point. If the organization is in a highly stable, mature industry, traditional hierarchical methods for management and control may be perfectly adequate and the requirements for adaptability, flexibility and empowerment might not be very high [Galbraith95]. Ceteris paribus, we believe that adaptability and flexibility are always desirable system attributes. Control may or may not be desirable. For example, in decision support situations, monitoring of the decision process could seriously inhibit the decision makers. Similarly, empowerment may or may not be a desirable design goal depending on the situation. For example, in an (administrative) workflow application for a mail-room one 
would probably concentrate on the throughput and control dimensions and leave very little freedom for the exercise of individual initiative.

\subsection{Mechanistic Design Variables}

In addition to the work/organizational requirements analysis described above, business processes can be characterized on a number of other dimensions that have important implications for their design. In particular, we identify five important dimensions: topological complexity, dynamic complexity, external connections/media, variability and throughput. We describe each of these dimensions in terms of a number of component variables that should be identified during the design process.

\section{Topological Complexity}

Topological complexity measures the shape, size and density of interconnections in the workflow system. High values for the following variables can be expected to increase the complexity of the workflow system making it harder to design, support and modify.

- Number of Agents: The number of agents involved in a workflow process is an indication of process complexity. For instance, workflow processes in a credit card management office with two hundred workers are likely to be much more complex than in another similar office with 10 workers.

- Number of Roles: The same number of agents may result in different complexities due to different number of agent types, i.e., roles. For instance, a bank with 100 agents is less complex than a hospital with 100 agents because hospital personnel are more specialized and will have more roles.

- Organization of Agents: Another important distinguishing factor is the way agents are organized. For example, complexity will increase if a relatively large number of formal organizational roles are needed for the purposes of approval, consultation, inspection, and so on.

- Number of Steps in the Workflow Process: The length of a workflow process in terms of the average and maximum number of sequential steps that need to be executed to process a given job or case.

\section{Dynamic Complexity of Workflow Processes}

The following temporal dimensions also have important implications for the design of the workflow process and the choice of supporting technologies.

- Interactions between Agents: Different cultures in similar organizations may result in different ways and frequencies that agents interact with each other during the workflow process. Some of these interactions may be process related, others may result from the need for socialization and learning. 
- Variability of Agent Composition: Some workflow may be ad hoc because its agent composition may change from time to time. For instance, a volunteer organization such as a blood donation drive committee may be composed of different agents for each workflow cycle

- Number of Exceptional Cases: Enumerating all possible combinations of events is impossible in practice. Therefore, systems are usually designed to handle the most frequently occurring events while known but rare events are handled as exceptions. The ability to handle entirely unanticipated events is a human quality which the workflow system should recognize and support.

- Duration of the Workflow Cycle: Some workflow processes may be completed in a matter of minutes while others may take days or months to complete. An example of the former is a lab test process of a blood sample; an example of the latter is a long running law suit.

- $\quad$ Repetitiveness of the Workflow Process: Many workflow processes only occur continuously, others only once in a while. An example of the latter is a Christmas charity organization that organizes volunteers to do some charity work once a year.

High values for the number of interactions, agent variability, exceptions and length of the work flow cycle are likely to imply higher procedural complexity. The other two variables may or may not add to the complexity of the workflow system depending on the capabilities of the particular WFMS which is chosen.

\section{External Connections/Media}

The nature of the connections to external systems/organizations and the number and types of the different media obviously has a strong influence on the type of WFMS that will be required and the design of the underlying workflow system. The reference model developed by the Workflow Management Coalition [WfMC96] specifies five interfaces to the central "Workflow Enactment Engine" to: Process Definition Tools, Administration and Monitoring Tools, Other Workflow Engines, Applications invoked by Workflow Clients and Applications invoked directly by the WFMS. For our purposes, the last three interfaces best reflect external connection requirements. Of particular relevance will be required connections to imaging systems, telephone call centers, mainframe systems, remote sites, the internet or intranet, and possible EDI applications. With regard to media, application requirements range from primarily text, to image, compound and multimedia documents, and possibly to voice and even video services.

\section{Process Variability}

Business processes can change either dramatically or incrementally over time. Understanding the sources and natures of process changes is important to make workflow system more adaptive to changes. 
- Organizational Redesign: Organizations may undertake major changes for many reasons such as merger and acquisition, major business reorientation, or business reengineering. The effect of organizational redesign can be dramatic as major changes in the grouping and the structure of work can occur [Mackenzie86].

- Procedure Adjustment: Changes in the way work is done can occur when business policies are adjusted in terms of decision making rules and management procedures. For instance, the approval procedures may change in a loan management system in order to tighten the loan amounts or the number of loans. This can occur when the economy shows a down turn and the loan provider (such as a bank) may want to protect itself from bad loans.

- Process Improvement and Innovation: In order to reduce production or service cycles, improve quality, and reduce costs, business process may be modified or completely redesigned without any dramatic change in the organizational structure [Davenport93].

- Interorganizational Relationship Changes: Interorganizational relationships can change over time either in the form of adding new or removing old organizations, or in terms of interaction procedures.

- Unanticipated Events: No matter how carefully a workflow management system is designed, there will always be unanticipated events or unsupported future events. A good system should be able to handle surprises without interrupting normal operations.

\section{Throughput and Responsiveness}

The requirements for high throughput and efficiency, particularly in value-added transaction-based processes, can impact the choice of WFMS and the resulting AWCSE properties. Flexible, LAN-based systems with desirable user features and a broad range of organizational capabilities as we have defined them, may not scale-up for use in major production systems, requiring the organization to build their workflow system around a mainframe (an example is the Fedex imaging system described in [Candleretal96]. In data processing, the number of transactions to be processed per unit time is often a good measure of throughput requirements. However, the concept of a "transaction" does not fit the multifaceted nature of work addressed by WFMS. Some possible metrics relating to throughput and responsiveness requirements are as follows:

- Cases per hour and total processing time per case: where a case is a unit of work such as a job or an extended transaction for a customer.

- Customer Response Time: average, minimum and maximum time taken to respond to a customer request. 


\subsection{Mechanistic Characterization of Business Processes}

For ease of reference, we will refer to the design variables defined in this section as the "CEVT" variables (topological and dynamic complexity, external connectivity/media, variability and throughput). By way of illustration, Table 5 shows possible requirements of each of the above process types in terms of the CEVT design variables.

\begin{tabular}{|l|l|l|l|l|l|}
\hline Process Type & $\begin{array}{l}\text { Topological } \\
\text { Complexity }\end{array}$ & $\begin{array}{l}\text { Dynamic } \\
\text { Complexity }\end{array}$ & $\begin{array}{l}\text { External/ } \\
\text { Media }\end{array}$ & $\begin{array}{l}\text { Process } \\
\text { Variability }\end{array}$ & $\begin{array}{l}\text { System } \\
\text { Throughput }\end{array}$ \\
\hline rdrbAccounting & High & Low & Low & Low & High \\
\hline Core & Depends & Medium & Depends & Depends & High \\
\hline Administrative & Medium & Medium & Low & Medium & High \\
\hline Management & Medium & Medium & Medium & Medium & Medium \\
\hline $\begin{array}{l}\text { Knowledge } \\
\text { intensive }\end{array}$ & Low & Medium & High & High & Low \\
\hline Ad hoc & Low & High & Low & High & Low \\
\hline
\end{tabular}

Table 5. Characterization of Business Processes with CEVT Design Variables

For example, the accounting process type is characterized by high topological complexity and low dynamic complexity as it usually involves many agents in the accounting department and is quite stable. An Accounting system is usually internally focused and requires only text and numeric data so that it has low requirements with regard to external connections and media. However, it may require high throughput capacity to handle large numbers of transactions. At the other extreme, an ad hoc process has most of its system requirements reversed in comparison to accounting processes. Core processes have variable requirements on topological complexity and process variability depending on the specific process and are therefore marked as "depends" in the table. For instance, customer service typically has low topological complexity while engineering design is more complex in topology. Note that the values in the characterization table are suggestive only and that it will require analysis of specific organizations and processes to develop more concrete values.

The design variables illustrated for hypothetical processes in Tables 4 and 5 provide some idea of the complexity of workflow systems. Further research will be needed to refine these concepts and to determine their usefulness in characterizing specific process design situations.

\section{Design Variables and Research Challenges}

In this section, we review the design variables that we have identified and indicate some of their implications for workflow system design and future research (see Table 6). 


\begin{tabular}{|l|l|}
\hline Organizational/Organic (AWCSE) & Process/Mechanistic (CEVT) \\
\hline Adaptability & Topological Complexity \\
\hline Worker Flexibility & Dynamic Complexity \\
\hline Control & External Connections/Media \\
\hline Organization Structure & Process Variability \\
\hline Empowerment & Throughput and Responsiveness \\
\hline
\end{tabular}

Table 6. Workflow System Design Variables

The organizational/organic variables in Table 6 come from our discussion in section 4 concerning the organizational and behavioral issues that we believe are of the utmost importance to the successful installation of BPA technologies. The Process/Mechanistic variables were discussed in section 5 and represent our attempt to provide a general characterization of important differences between business processes that are likely to impact the design and selection of BPA software.

Adaptability measures the difficulty and expense of changing the workflow system in response to a change in external conditions or management needs. Adaptability is high when unforeseen changes in the structure of the process or the requirements for its execution can be accommodated quickly and cheaply. (Legacy systems based on mainframe technologies are notorious for their low adaptability.) Note that adaptability in the organizational sense is similar to the implied need for adaptability at the process level implied by high process variability. However, adaptability is a more general concept as it applies across all the processes of the organization. To achieve adaptability at the organizational level, will require a common language and set of hardware and software standards. "Reference models" which show standard configurations and functionalities for the various classes of workflow system shown in table 4 , could help in this regard. Finally, we need to understand the socio-technical aspects of workflow automation and how a culture of change and flexibility can be encouraged at an organizational level, [Mumford\&Beekman94].

Work Flexibility measures the amount of freedom that users of the system have to take actions and exercise unplanned options that follow common sense rather than the dictates of a preplanned step-bystep procedure. A major function of a workflow management system is to route tasks in a certain sequence among agents who perform different roles. These routing schedules should be maximally flexible consistent with getting the job done. A normal routing map can be used to pass the tasks based on certain events and conditions. However, exceptions and rework are difficult to support using simple routing maps; on the other hand, routing sequences must also be controlled. One solution is to allow dynamic routing so long as given sequence constraints associated with each document class are obeyed [Kumar\&Zhao96]. An alternative approach offered by one WFMS vendor is to enable workflows to occur initially with no routing 
constraints; if definite patterns of use emerge after a period of use, they can then be formalized in the form of work routings [CSE95].

As discussed in section 4, most work is not simply a mechanical process that can easily be captured by rules, worklists, routing schedules and known procedures. Rather, there is a need to rely on human judgment and problem solving capabilities. Research on how exceptions and "work-arounds" to the workflow system can be accomodated and, on occasion, encouraged, could be useful in achieving higher success rates in BPA projects. This means, for example, that a well designed workflow system might encourage rather than inhibit human contact. Provision for richer mediums of communication between team members such as telephone or even video conversations might be needed in problem solving tasks for example.

Control is a third process variable that has strong influence on the design of the workflow system. There are two types of control. The first concerns the need for fiduciary responsibility, accountability, auditability and security that occurs if the process involves financial transactions or highly confidential information or is one of the mission critical core processes of the enterprise. Developing secure and safe workflow systems is an active area of research in WFMS and active databases [Ellmer94, Tewari94]. Because WFMS maintain audit trails and can ensure that appropriate authorizations are obtained and separation of duties is maintained, WFMS could have an important role in risk management systems. Research is needed on how optimally to allocate access and performance rights to individuals, teams and roles so that work performance is optimized for a given degree of risk .

The second type of control is managerial and focuses on monitoring the state of the organization and evaluating the performance of departments, teams and individuals. BPA technologies provide managers with the ability to monitor the state of the organization in the minutest detail. However, as illustrated in Section 4, this is not always desirable. Workflow system designers need to exercise discretion with regard to the collection and distribution of data and its use for performance evaluation. WFMS systems should be capable of collecting and presenting data at either the individual or team level. The presentation of "scripts" detailing the steps to be taken by human operators when dealing with customers or performing tasks is another capability of WFMS that bears on issues of organizational control. Research is needed on the impact of various types of automated monitoring and management control on work productivity.

Organization Structure refers to the allocation of decision authorities, the delineation of roles, the formal recognition of vertical and horizontal reporting relationships and the existence of policies and rules for managing work. In a team-based organization, the roles and responsibilities of teams are also important structural components. While formal hierarchies may not be observed rigorously in actual organizations, it 
is nevertheless necessary for the WFMS to be able to represent them. This is obviously the case in processes involving approvals and signatures by managers but it will also be true in other cases where referrals to higher authorities due to unforeseen or exceptional cases are necessary. The challenge for WFMS is to represent structure in a manner which is readily visible and changeable by management as required. Perhaps an even greater challenge is to enforce these formal relationships in a manner which is as flexible and human as possible. On the technical side, research is needed on rule-based techniques that can store structural information in a form that can be maintained without resort to system programming. The Event-Condition-Action (ECA) rules in active databases [Hanson92] should be extended into Event-RoleObject-Condition-Action (EROCA) rules with the addition of role and object clauses [Kumar\&Zhao96]. These two clauses are necessary in workflow management because authorizations and controls require the knowledge of the roles and the documents. They are not needed in a database environment because the authorizations and operations in databases are relatively simple and the data elements are not document dependent.

Empowerment measures the ability of users to make substantive decisions without approval from higher authority and to exercise design control over the work process itself [Hammer\&Champy93]. The extent to which workers are empowered is an organizational design choice. Modern, "organic" forms of organization tend to have high levels of empowerment, [Womacketal90]. To empower workers means that the WFMS must support human decision making rather than replace it. It also means that the workers themselves should have the ability to redesign their work processes as necessary. This is a challenge for the designers of such systems since any redesigned system must maintain the integrity of the underlying process.

Topological and dynamic complexity as defined above contribute to the overall procedural complexity of a business activity. If a process has high procedural complexity it is likely to be difficult to develop the workflow system, to operate it without an extended training period, and to modify it to meet the changing needs of the enterprise. Understanding and measuring the complexity of workflow systems is an important issue as it can guide project design choices and help in the estimation of project costs and risks.

External Interfaces/Media: This design variable raises many technical questions and will have a strong influence on the choice of WFMS. The major technical research issues are concerned with the evolution of standards such as the OMG Group's CORBA standard for interchanging complex objects. On the managerial side, the major research issues concern the integration of the WFMS with other systems and internal and external organizations. Research is also needed with regard to the usefulness of different media 
in various work situations and whether multiple media (e.g. synchrounous data and voice communications) can increase work productivity and quality.

Procedural variability indicates how frequently changes may occur in the supported business procedure. High variability implies frequent changes in the process and the need for the underlying software to be very adaptable. At the implementation level, object-oriented programming techniques and object-oriented database management systems can be used to implement flexible workflow management systems to cope with frequently changing requirements in an organization [Kappe195]. In principle, objectoriented technology should facilitate the customized design of workflow systems to meet the needs of specific business processes and to allow dramatic changes in organizational redesign and process innovation. Another area for research is to develop version management techniques (based on temporal logic) so that changes in work processes can be captured and properly synchronized between different versions of processes. Version management has been well studied in the context of data management [Won\&Elmasri96]. However, version management in workflow management systems is more complex than in database management systems because WFMS involve the storage of not only data but also procedures, roles, and agents.

Stringent throughput and response requirements may limit the choice of WFMS. To the extent that there is a trade-off between functionality and speed of execution, some of the advanced features and GUI front-ends of advanced WFMS may have to be sacrificed. Human capabilities for work have been studied since the dawn of scientific management and the transaction throughput capabilities of information processing systems are well documented. Research is needed on the combined information and decision processing capability of man-machine systems such as WFMS.

The organic AWCSE and mechanistic CEVT design variables summarized in this section are a first attempt at developing a systematic approach to designing workflow systems and selecting the desired features in a WFMS. We believe that any adequate design procedure should consider all of these variables both within the context of total organization needs and in the context of specific process redesign.

The above discussion also indicates many important research directions. As workflow systems can touch the very core of the organization and impact every facet of working life, it will be important not only to study the specific issues mentioned above, but also to undertake surveys and field studies of the total impact of workflow automation on organizations. 


\section{Conclusion}

BPA technologies and WFMS in particular, have the potential to alter the nature of work in organizations. While they are probably evolving satisfactorily towards a rational "mechanistic" representation of work and business processes, they will also need to satisfy the more "organic" needs of work and organizations. As management changes their view of the objectives and structure of the organization, WFMS must fit seamlessly into whatever organizational philosophy is needed to further the aims of the organization.

The Triangular BPA Adaptation Model in Section 3 emphasizes the dual requirements for work and organizational fit and specific process fit. From an analysis of the need to fit organizational and work related needs, we developed a number of variables that we believe must be considered in the design of any workflow system. The AWCSE requirements, namely adaptability, work flexibility, control, structure and empowerment as defined in section 4, represent the "organic" side of organizations. With regard to the need for the WFMS to fit the needs of a specific process, we developed a number of variables in section 5 , that measure the more mechanistic dimensions of business processes. These CEVT variables (procedural complexity, external connections/media, variability and throughput), along with the AWCSE variables, provide a basis for a general approach to the design of work flow systems and the selection of WFMS. By classifying and analyzing common classes of business process, we demonstrated that different processes can have very different values for both the AWCSE and CEVT variables. Consequently, different types of process are likely to demand different process automation technologies and workflow management systems.

We believe that the future will see variations of workflow integration methodologies and systems, each of which will focus on providing automation solutions to one or more types of business process. In the longer-run, these separate workflow systems will integrate all the other BPA technologies and will evolve into a single entity which will be central to the survival of the enterprise. Careful consideration of the implications of the design variables identified in this paper should guide this future evolution. The design variables and associated research directions listed in Section 5 and the measures to achieve software adaptability discussed in section 6 indicate many possible directions for future research.

We are currently working toward a methodology for BPA system design and evaluation that is based on the model and design variables developed in this paper. This methodology should provide useful guidelines at both the system planning level and the system design level.

\section{References}

[Aiken\&Hage68] Aiken, M. and J. Hage, "Organizational Independence and Intra-Organizational Structure”, American Sociological Review, Vol 33 (December, 1968), pp. 912-930. 
[Andresen95] Andresen, Gary L., "Business Process Automation -- Transforming the Enterprise by Automating Access to Information, Customers, and Communications", White Paper, Dataquest, Inc., June 1995.

[Burns\&Stalker61] Burns, Tom and G. M. Stalker, The Management of Information, Tavistock: London, 1961.

[Bussler\&Jablonski94] Bussler, Christoff and Stefan Jablonski, "An Approach to Integrate Workflow Modeling and Organization Modeling in an Enterprise", Proc. Third Workshop on Enabling Technologies: Infrastructure for Collaborative Enterprises, IEEE Computer Society Press, Morgantown, WV, 1994.

[Candlereta196] Candler, James W., Prashant C. Palvia, Jane D. Thompson and Steven M. Zeltmann, "The ORION Project: Staged Business Process Reengineering at FedEx", Communications of the ACM, Vol.39, No. 2, February 1996, pp. 99-107.

[CSE95] "WorkFlow Management: An Introduction", CSE Systems, Computer \& Software Engineering Ges.m.b.H., Klagenfurt, Austria, 1995.

[Davenport93] Davenport, Thomas H., Process Innovation: Reengineering Work though Information Technology, Harvard Business School Press, 1993.

[Davenport\&Stoddart94] Davenport, Thomas H. and Donna B. Stoddart, "Reengineering Business Change of Mythic Proportions", MIS Quarterly, June, 1994, pp. 121-127.

[Deshpande82] Deshpande, Rohit, “The Organizational Context of Market Research Use”, Journal of Marketing, Vol 46 (Fall 1982), pp. 91-101.

[Ear194] Earl, Michael J., "The New and the Old of Business Process Redesign", Journal of Strategic Information Systems, Vol 3, No. 1, 1994, pp. 5-22.

[Ellmer94] Ellmer, E.; Pernul, G.; Quirchmayr, G. "Security for workflow management". Proceedings of the Sixth International Conference on Parallel and Distributed Computing and Systems, Washington, DC, USA, 3-5 Oct. 1994, pp. 257-60.

[Galbraith95] Galbraith, Jay R., Designing Organizations, Jossey-Bass Inc, 1995.

[Georgakopoulos95] Georgakopoulos, Dimitrios, Mark Hornick, and Amit Sheth, "An Overview of Workflow Management: From Process Modeling to Workflow Automation Infrastructure”, Distributed and Parallel Databases, Vol. 3, No. 2, pp. 119-153, 1995.

[Grovereta195] Grove, Varun, Syung Reol Jeong, William J. Kettinger, and James T. C. Teng, "The Implementation of Business Process Engineering", Journal of Management Information Systems, Vol 12, No 1, Summer 1995, pp. 109-144.

[Halletal67] Hall, R., E. F. Haas and N. F. Johnson, "Organizational Size, Complexity and Formalization", American Sociological Review, Vol 32 (December, 1967), pp. 903-911.

[Hammer\&Champy93] Hammer, Michael and James Champy, Reengineering the Corporation: A Manifesto for Business Revolution, Harper Business, 1993.

[Hanson92] E. N. Hanson, "Rule Condition Testing and Action Execution in Ariel", Proceedings of the 1992 ACM SIGMOD International Conference on Management of Data, June 1992.

[Hewitt86] Hewitt, Carl, “Offices are Open Systems", ACM Transactions on Office Systems, Vol 1, No 3, July 1986, pp. 271-287.

[Kappe195] Kappel, G., P. Rausch-Schott, S. and W. Retschitzegger, "TriGS: Active object-oriented workflow management", Proceedings of the Twenty-Eighth Hawaii International Conference on System Sciences, Wailea, HI, USA, 3-6 Jan. 1995.

[Sproull\&Kiesler91] Sproull, L. and S. Kiesler, Connections: New Ways of Thinking in the Networked Organization, MIT Press, Cambridge, Mass., 1991. 
[Kumar\&Zhao96] Kumar, Akhil and J. Leon Zhao, “A Framework for Dynamic Routing and Operational Integrity Controls in a Workflow Management System", Proceedings of the 29th Annual Hawaii International Conference on Systems Sciences, January, 1996.

[Lai88] Lai, Kum-Yew, Thomas W. Malone and Keh-Chiang Yu, "Object Lens: A 'Spreadsheet' for Cooperative Work", ACM Transactions on Office Systems, Vol 6, No 1, October 1988, pp. 334 - 353.

[Lee88] Lee, Ronald M., "Bureaucracies as Open Systems", ACM Transactions on Office Systems, Vol 2, No 6, April 1988, pp 271-287.

[Lochovsky83] Lochovsky, Fred H., "Improving Office Productivity: A Technology Perspective", Proceedings of the IEEE, Vol. 71, No. 4, April 1983, pp. 512-518 (Invited Paper).

[Lucas95] Lucas, Henry C., The T-Form Organization: Using Technology to Design Organizations for the $21^{\text {st }}$ Century, Jossey-Bass: San Francisco. 1995.

[Mackenzie86] Mackenzie, Kenneth D., Organizational Design: The Organizational Audit and Analysis Technologies, Ablex Publishing Corporation, 1986.

[Mintzberg79] Mintzberg, H., The Structuring of Organizations, Englewood Cliffs, NJ:Prentice Hall, 1979.

[Mumford\&Beekman94] Mumford, E., and J-G. Beekman, Tools for Change: A Socio-Technical Approach to Business Process Reengineering, CSG Publications, 1994.

[Orlikowski92] Orlikowski, W., "Learning from Notes: Organizational Issues in Groupware Implementation", Technical Report \#131, Center for Coordination Science, MIT, Cambridge, MA, 1992.

[Orlikowski\&Robey91] Orlikowski, W. J.and D. Robey, "Information Technology and the Structuring of Constraints", Information Systems Research, Vol. 2, No. 2, June 1991. pp. 143-169.

[Ouchi79] Ouchi, William G., "A Conceptual Framework for the Design of Organizational Control Systems", Management Science, Vol 25, 1979, pp. 833-848.

[Pike\&Barnes94] Pike, John and Richard Barnes, TQM in Action, Chapman and Hall: London.1994.

[Sachs95] Sachs, Patricia, "Transforming Work: Collaboration, Learning, and Design", Communications of the $A C M$, Vol.38, No. 9, September 1995, pp. 36-45.

[Stein\&Zwass95] Stein, Eric W. and Vladimir Zwass, "Actualizing Organizational Memory with Information Systems”, Information Systems Research, Vol.6, No. 2, 1995, pp. 85-117.

[Stoddart\&Jarvenpaa95] Stoddart, Donna B., and Sirkka L. Jarvenpaa, "Business Process Redesign: Tactics for Managing Radical Change”, Journal of Management Information Systems, Vol 12, No 1, Summer 1995, pp. 81109.

[Suchman83] Suchman, L., "Office Procedures as Practical Action: Models of Work and System Design”, ACM Transactions on Office Systems, Vol. 1, No. 4, October 1983, pp. 320-328.

[Tewari94] Tewari, H., McCourt, M., and O'Mahony, D. “Advanced electronic commerce security in a workflow environment". Electronic Commerce. Current Research Issues and Applications, Gaithersburg, MD, USA, 1 Dec. 1994, pp. 69-85.

[Wastelleta194] Wastell, David G., P. White and P. Kawalek, "A Methodology for Business Process Redesign: Experiences and Issues", Journal of Strategic Information Systems, Vol 3, No 1, 1994, pp. 23-40.

[WfMS96] http://www.aiai.ed.ac.uk/WfMC/index.html.

[Womacketal90] Womack, James P., Daniel T. Jones, and Daniel Roos, The Machine that Changed the World: The Story of Lean Manufacturing, Harper Collins, 1990.

[Won\&Elmasri96] Won, Jongho and Elmasri, R. "Representing Retroactive and Proactive Versions in Bitemporal Databases (2TDB)", Proceedings of the Twelfth International Conference on Data Engineering, New Orleans, LA, USA, 26 Feb.-1 March 1996, pp. 85-94. 